injury to the midportion of the cerebellum with dentate nuclear involvement, the greater the risk of developing post-operative mutism (Dietze DD Jr., Mickle JP. Cerebellar mutism after posterior fossa surgery. Pediatr Neurosurg; Oct 1991; 16:25-31). (Reprints: Donald D. Dietze, Jr., M.D., Department of Neurosurgery, University of Florida, Box J-265, J. Hillis Miller Health Center, Gainesville, FL 32610.)

COMMENT. The authors advise that this possible complication of posterior fossa surgery should be discussed with patient and parents as part of the preoperative planning. Mutism is not restricted to tumor pathology but may be associated with any acute pathological insult to the midportion of the cerebellum. Fortunately, the mutism resolves completely or to a mild cerebellar dysarthria over a 3 month period. Surgery of midline medulloblastomas is particularly at risk for the development of postoperative mutism.

\title{
MANAGEMENT OF INTRACRANIAL NEOPLASMS IN NEUROFIBROMATOSIS
}

Recommendations are proposed for the diagnosis and management of CNS neoplasms with NF-1 and NF-2 at the Department of Neurology, Section of Pediatric Neurology, Cleveland Clinic Foundation, Cleveland, Ohio; Phoenix Children's Hospital, Phoenix, AZ; and Children's National Medical Center, George Washington University, Washington, D.C.: (1) MRI is indicated in most childhood patients with NF-1 to screen for visual pathway astrocytomas with substantial visual loss or progressive disease. Treatment should include surgery, irradiation or chemotherapy. (2) Focal areas of signal intensity identified in asymptomatic patients should not be treated as neoplastic unless there is pathologic confirmation. (3) Specific CNS neoplasms (i.e., astrocytomas, ependymomas, meningiomas) should be treated with standard management protocols. (4) Intrinsic brainstem tumors with NF1 should be observed for progressive disease and considered for biopsy and/or resection. (5) Gadolinium-enhanced MRI for acoustic neuromas is indicated in children at risk for NF-2. (6) Formal protocols to clarify the incidence, natural history, and management of intracranial neoplasms in NF-1 and NF-2 should be developed in collaborative national studies (Cohen BH, Kaplan AM, Packer RJ for the Children's Cancer Study Group. Management of intracranial neoplasms in children with neurofibromatosis type 1 and 2 . Pediatr Neurosurg Nov 1991; 16 :66-72). (Reprints: Availability and source not

COMMENT. Optic nerve and tract astrocytomas are the most common intracranial neoplasms in NF-1 with an incidence of 5-15\%. They may present with proptosis, extraocular nerve palsies or nystagmus. Some patients have MRI appearance of "tumor streaking" without mass effect, from the chiasm along the optic tracts and optic radiations. The significance of this streaking is not known. The guidelines for the treatment of visual pathway tumors is controversial, especially for asymptomatic patients or patients with non-progressive defects. For an overview of CNS tumors and neurofibromatosis see Progress in Pediatric Neurology, Millichap ed. 1991, pp. 374-379. 